\title{
Las aulas virtuales: una herramienta de apoyo y seguimiento al proceso de enseñanza y aprendizaje en los cursos presenciales de Lectoescritura de la USTA
}

\author{
Clara Inés Jaramillo Gaviria ${ }^{1}$. \\ Carlos Eduardo Álvarez Martínez².
}

El hombre es un animal hablador, porque capta, lee, elige el interior de las cosas, el más allá de su apariencia inmediata. Porque entiende y elige, es capaz de hablar y de razonar y de muchas otras actividades... La pa labra es y será siempre el vehículo de la realidad. Sólo cuando existan las palabras, el hombre comprenderá su mundo y se experimentará como un ser situado en él. Santo Tomás de Aquino.

Para adaptarse a las necesidades de la sociedad actual, las instituciones de educación superior deben flexibilizarse y desarrollar vías de integración de las tecnologías de la información y la comunicación en los procesos de formación. Paralelamente es necesario aplicar una nueva concepción de los alumnos-usuarios, así como cambios de rol en los profesores y cambios administrativos en relación con los sistemas de comunicación y con el diseño y la distribución de la enseñanza. Todo ello implica, a su vez, cambios en los cánones de enseñanza-aprendizaje hacia un modelo más flexible. Para entender estos procesos de cambio y sus efectos, así como las posibilidades que para los sistemas de enseñanza-aprendizaje conllevan los cambios y avances tecnológicos, conviene situarnos en el marco de los procesos de innovación. Jesús Salinas

\begin{abstract}
Resumen.
Desde la experiencia de los cursos de lectoescritura en la USTA, podemos ratificar la idea de que la modalidad virtual no es opuesta a la presencial. Las aulas virtuales articulan los conceptos y destrezas aprendidas en las horas de trabajo presencial con las horas de trabajo independiente del estudiante. Las aulas virtuales permiten repasar, relacionar y reforzar los contenidos para luego ser aplicados en el contexto de la Educación Superior a través de ejercicios y lecturas especializadas. De la misma forma es necesario que los docentes trabajen en equipo con la oficina de Educación Virtual y sean asesorados por esta, con el fin de hacer un seguimiento del uso real de las aulas virtuales e implementar planes de mejora que enriquezcan los procesos de enseñanza y aprendizaje.
\end{abstract}

Palabras clave: Trabajo independiente del estudiante, Ambientes Virtuales de Aprendizaje (AVA), Objetos Virtuales para el Aprendizaje (OVA), aula virtual, lectoescritura, enseñanza y aprendizaje, Educación Superior.

\begin{abstract}
.
The analysis of the results provided by the Reading and Writing Courses offered by the USTA demonstrates that v-learning is not opposed to classroom-learning. Virtual environments link concepts and skills learned through traditional methods with independent individual work. Vlearning allows to review and relate different types of knowledge which can later be applied in the context of higher education. Teachers must work shoulder to shoulder with the V-learning bureau in order to track, on real time, the use of virtual environments by the students in order to improve teaching and learning processes.
\end{abstract}

Key words: Virtual literacy, independent study, virtual classroom, Learning Objects, Virtual Learning Environments.

En los últimos años, las instituciones de educación superior han implementado cursos de lectoescritura con el fin de mejorar las competencias comunicativas (lingüísticas) de los estudiantes y profesores. Para cumplir con este objetivo, la Universidad Santo Tomás, desde el año 2013, creó la Coordinación en Lectoescritura adscrita al Departamento de Humanidades y Formación Integral, y con el apoyo de la Oficina de Educación Virtual implementó los Ambientes Virtuales de Aprendizaje (AVA) como herramienta didáctica

\footnotetext{
${ }^{1}$ Magister en Educación. Coordinadora Lectoescritura - Departamento de Humanidades y Formación Integral, Universidad Santo Tomás.

${ }^{2}$ Magister E-Learning. Magister en Ingeniería de Sistemas. Coordinador Oficina de Educación Virtual Universidad Santo Tomás.
} 
para el Trabajo Independiente de los estudiantes (T.I.). En este sentido, las estrategias virtuales -como Objetos Virtuales para el Aprendizaje (OVA), instrumentos de evaluación, foros, chat, interacción e interactividad, entre otros- han innovado y enriquecido los procesos de enseñanza y aprendizaje de lectoescritura.

El propósito de esta ponencia es presentar los aspectos relevantes y problemáticos de la experiencia del uso de Ambientes Virtuales de Aprendizaje como herramienta de apoyo para las horas de trabajo independiente de los estudiantes en los cursos presenciales de lectoescritura en la Educación Superior. La siguiente pregunta servirá de hilo conductor para esta reflexión: ¿Cómo promover el uso de los Ambientes Virtuales de Aprendizaje como herramientas de apoyo para el trabajo independiente de los estudiantes en los cursos de lectoescritura de los programas de la modalidad presencial? Frente a esta pregunta, queremos defender la siguiente tesis: la virtualidad no es opuesta a la modalidad presencial, pues el uso de Ambientes Virtuales de Aprendizaje, como herramientas de apoyo para el trabajo independiente, enriquece y complementa los procesos educativos presenciales; no obstante, las instituciones de Educación Superior y la comunidad académica (directivos, profesores y estudiantes) todavía no generan las condiciones de posibilidad para su óptimo desarrollo, ya que su énfasis principal sigue orientado hacia la modalidad presencial tradicional.

La Escuela Progresista o Escuela Nueva, que surgió a mediados del siglo pasado, planteó la reflexión sobre la necesidad de orientar la educación hacia la democracia y la libertad, con el fin de formar ciudadanos que transformarán la realidad social a partir de nuevas metodologías de enseñanza y aprendizaje, fundamentadas en el aprender haciendo (learning by doing). Así, dicha escuela, intentaba romper con el esquema tradicional de la lección magistral que se caracterizaba por ser un proceso de comunicación unidireccional entre el profesor que transmite la información y los alumnos como receptores pasivos.

En 1970, la revisión a la Ley de Educación se convirtió en una demanda del Fondo Monetario Internacional (FMI) y del Banco Mundial (BM) para todos los países de Hispanoamérica; esto le implicó a la educación un giro competitivo que la orientó hacia la oferta de un mejor producto o servicio adecuado a las exigencias propias de la globalización. Todos estos cambios se realizaron pensado en la formación de profesionales preparados para enfrentar las dinámicas que genera el modelo económico del conocimiento y así fomentar una ley de educación para "el trabajo". Al respecto, Sunkel afirma:

Los países de América Latina han realizado a lo largo de este tiempo importantes esfuerzos para no permanecer al margen de esta tendencia global. A finales de los 80 y principios de los 90 se comenzaron a gestar las primeras políticas y programas TIC orientados a las escuelas... En América Latina la vía fundamental para la integración de las TIC en la educación ha sido la política pública, principalmente a través de programas y proyectos. Aunque en la actualidad solo un tercio de los países de la región ha diseñado una política formal de TIC en educación, la mayor parte ha desarrollado iniciativas con el carácter de proyectos o programas y además cuenta con una unidad especializada en el Ministerio de Educación que es responsable de su implementación (Sunkel, 2011). 
En la conferencia mundial sobre la educación superior de la UNESCO (1998), se aprobó la “Declaración

mundial sobre la Educación Superior en el siglo XXI: visión, acción y marco de acción prioritaria para el cambio y el desarrollo de la educación superior". El Artículo 12 de este documento, titulado “El potencial y

los desafios de la tecnología”, indica:

Los rápidos progresos de las nuevas tecnologías de la información y la comunicación seguirán modificando la forma de elaboración, adquisición y transmisión de los conocimientos. También es importante señalar que las nuevas tecnologías brindan posibilidades de renovar el contenido de los cursos y los métodos pedagógicos, y de ampliar el acceso a la educación superior. No hay que olvidar, sin embargo, que la nueva tecnología de la información no hace que los docentes dejen de ser indispensables, sino que modifica su papel en relación con el proceso de aprendizaje, y que el diálogo permanente que transforma la información en conocimiento y comprensión pasa a ser fundamental. Los establecimientos de educación superior han de dar el ejemplo en materia de aprovechamiento de las ventajas y el potencial de las nuevas tecnologías de la información y la comunicación, velando por la calidad y manteniendo niveles elevados en las prácticas y los resultados de la educación, con un espíritu de apertura, equidad y cooperación internacional (UNESCO, 1998).

Los tres momentos referenciados anteriormente, plantean como lugar común un cambio de paradigma en el proceso de enseñanza y aprendizaje, donde el uso de los Ambientes Virtuales de Aprendizaje (AVA) se han constituido en herramientas idóneas para afrontar el mundo contemporáneo, el cual se caracteriza por el fomento del trabajo, la globalización y la sociedad del conocimiento.

Los Ambientes Virtuales de Aprendizaje (AVA) son espacios Web que posibilitan escenarios de interacción entre los actores del proceso formativo y los recursos didácticos. Se fundamentan en el aprendizaje cooperativo y colaborativo ya que los estudiantes participan y expresan sus inquietudes en los foros, chat, etc., y permiten que a través de las herramientas multimedia el docente produzca un entorno interactivo de construcción de conocimiento. Es decir,

Los ambientes virtuales de aprendizaje son aquellos que se implementan sobre redes virtuales, también llamadas redes conversacionales, que no son otra cosa que la concreción de relaciones, a través de la red, entre personas que tienen acceso a redes de computadores. A través de las redes conversacionales se pueden intercambiar ideas y compartir materiales, muy a menudo almacenados y presentados como redes de información multimedia (Gavils Panqueva, 1998, págs. 247-260).

Ahora bien, para implementar los Ambientes Virtuales de Aprendizaje en la Educación Superior se requiere: una infraestructura que permita el uso de las Tecnologías de la Información y la Comunicación (TIC); el acceso a sistemas de información locales y globales para consultar y compartir el conocimiento; la participación en las redes sociales y académicas que permiten difundir, debatir, compartir y apropiarse del conocimiento; y lo más importante, capacitar a la comunidad educativa (administrativos, docentes y estudiantes) con el fin de potenciar y optimizar los AVA en el quehacer académico cotidiano.

Además de lo anterior, para elaborar Ambientes Virtuales de Aprendizaje las Instituciones de Educación Superior requieren un equipo de trabajo especializado tal como: administrador del sistema, diseñador del sistema, desarrolladores multimedia, Webmaster, asesores pedagógicos, diseñadores instruccionales (educativo) y editores que apoyen el proceso de diseño, desarrollo, presentación e implementación. Es decir, que en asocio con los docentes, diseñe los Objetos Virtuales de Aprendizaje (OVA), las aulas virtuales, los 
videos, etc.; con el fin de que el campus virtual se alimente con la producción académica de secuencias didácticas y no sea un simple repositorio donde se suben recursos tomados de la Internet, como lecturas, videos, programas, syllabus, etc.

La Educación Superior hace uso de los AVA a través de tres modalidades, a saber:

\begin{tabular}{|c|c|c|}
\hline $\begin{array}{l}\text { MODALIDA PRESENCIAL } \\
\text { m-learning }\end{array}$ & $\begin{array}{l}\text { MODALIDAD A DISTANCIA } \\
\text { Blended Learning }\end{array}$ & $\begin{array}{l}\text { MODALIDAD VIRTUAL } \\
\text { e learning }\end{array}$ \\
\hline
\end{tabular}

Las tres modalidades, desde su especificidad metodológica, comparten la misma finalidad: la formación de profesionales. Lo que tienen en común es que el estudiante debe dedicar un tiempo determinado a sus actividades académicas. Este tiempo está dividido en: tiempo de trabajo dirigido y tiempo de trabajo independiente. Según el Ministerio de Educción Nacional (MEN):

El Crédito Académico equivale a 48 horas totales de trabajo del estudiante, incluidas las horas académicas con acompañamiento docente y las demás horas que deba emplear en actividades independientes de estudio, prácticas, preparación de exámenes u otras que sean necesarias para alcanzar las metas de aprendizaje propuestas (...) Por lo general, en asignaturas típicas, una hora de clase implica dos horas adicionales de trabajo independiente en pregrado y tres en posgrado (Ministerio de Educación Nacional, 2001).

Como vemos, el trabajo independiente es fundamental para el proceso de enseñanza y aprendizaje. No obstante, en la modalidad presencial se le hace poco seguimiento al proceso autónomo del estudiante. De ahí que consideremos que los AVA pueden constituirse en una herramienta complementaria para que el docente guíe el trabajo independiente de los estudiantes, con el fin de que profundicen en aquellos temas que, luego de trabajados en clase, presenten algún grado de dificultad teórica, práctica o requieran refuerzo.

Es decir, en la modalidad presencial se requiere que el docente identifique los temas que los estudiantes deben reforzar o practicar en las horas asignadas para su trabajo independiente y a partir de éstos diseñar recursos educativos digitales tales como: OVAS, glosario, material de apoyo, ejercicios de modelación, ejercicios de práctica, foros, chat, etc., que integrarán en el AVA. Luego que los estudiantes han interactuado con el AVA, el docente debe pedirles que en la siguiente clase socialicen el trabajo realizado con el fin de retroalimentar y aclarar las dudas antes de iniciar el proceso de evaluación. Este ciclo se explica en el siguiente gráfico. 


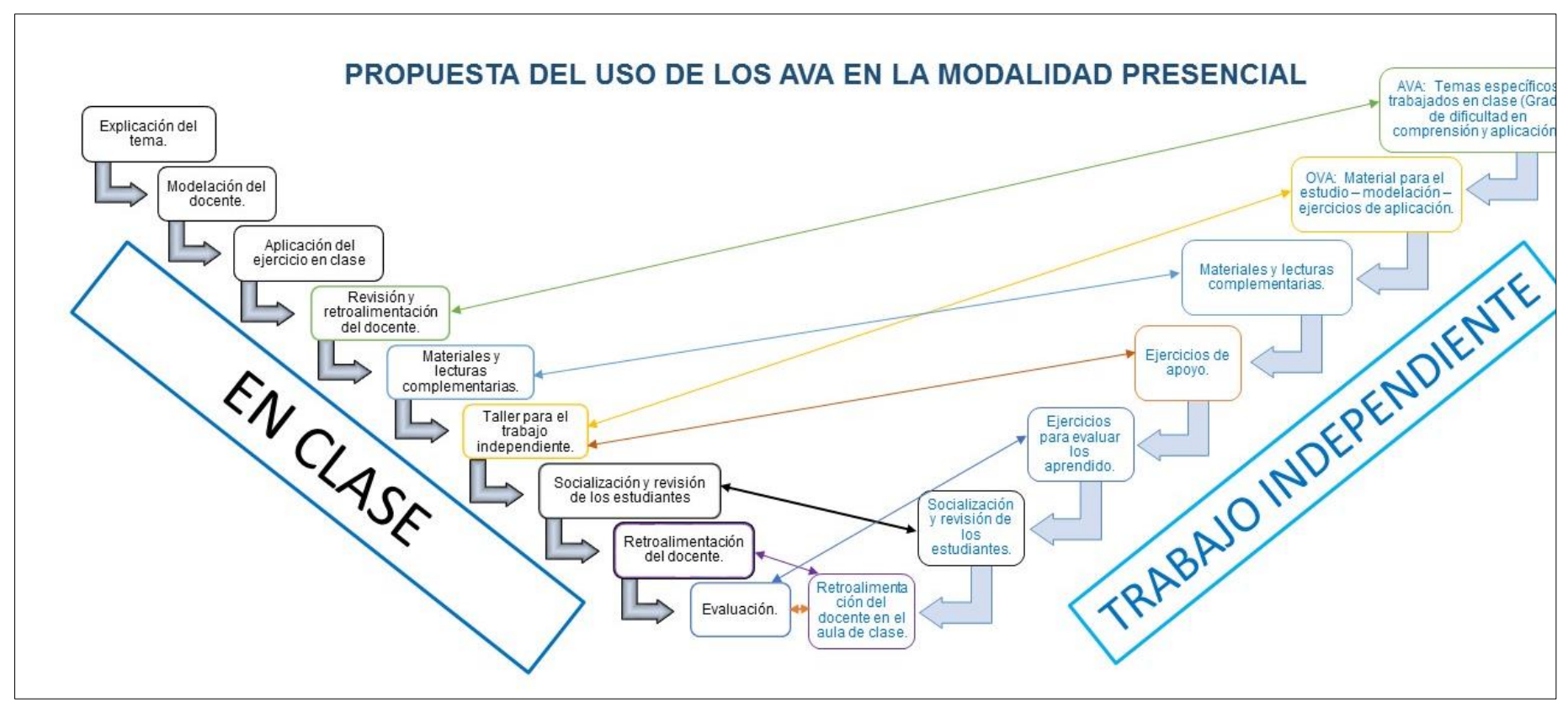

Ahora veamos cómo se ha implementado el uso de los AVA en los cursos de Lectoescritura que acompaña el Departamento de Humanidades y Formación Integral de la Universidad Santo Tomás en la sede de Bogotá.

A partir del análisis de los resultados de las pruebas ECAES (hoy SABER PRO), en lo que se refiere a las competencias de comprensión lectora y escritura, la USTA, a partir del año 2005, diseñó en su plan de mejoras los cursos de Nivelación en Lectoescritura para los estudiantes neotomasinos. En el año 2012, la Facultad de Ciencias y Tecnologías, adscrita a la Vicerrectoría de Universidad Abierta y a Distancia (VUAD), lideró un proyecto para implementar el Curso de Habilidades Comunicativas en modalidad virtual. En el año 2013, se creó la Coordinación de Lectoescritura que, con el apoyo de la Oficina de Educación Virtual, implementó los Ambientes Virtuales de Aprendizaje como herramienta didáctica para el trabajo independiente de los estudiantes en modalidad presencial. En este sentido, las estrategias virtuales que posibilitan la interacción e interactividad -como Objetos Virtuales para el Aprendizaje (OVA), instrumentos de evaluación, foros, chat, entre otros- han innovado y enriquecido los procesos de enseñanza y aprendizaje de lectoescritura,

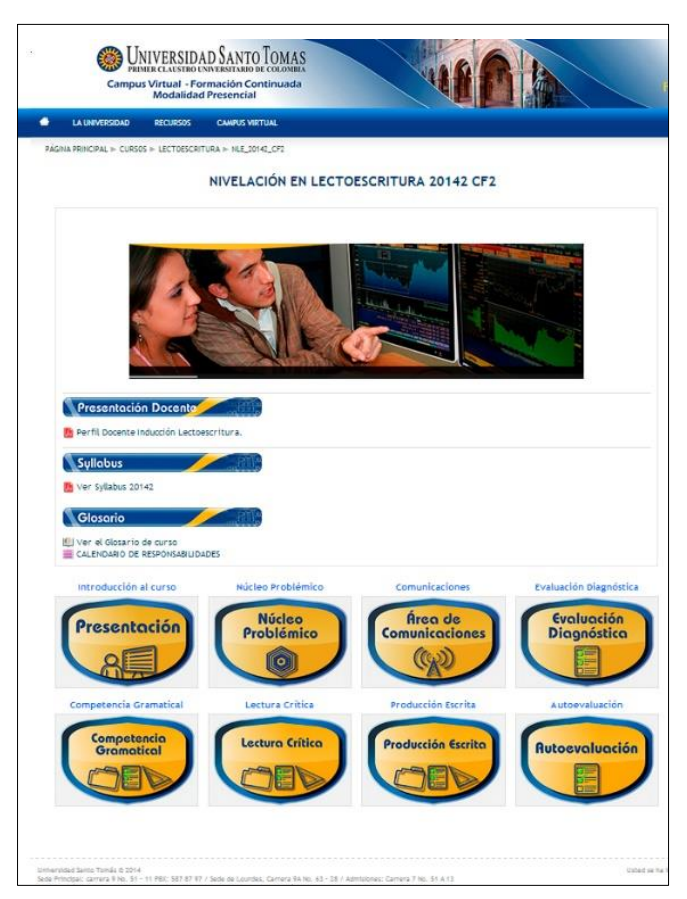
con la finalidad que los estudiantes fortalezcan sus habilidades comunicativas.

En los AVA se consolidó la propuesta metodológica del proceso de enseñanza y aprendizaje con el fin que tanto los docentes como los estudiantes, accedieran a los recursos para despejar dudas, practicar o repasar los temas durante o fuera de clase.

A continuación presentamos la estructura básica y funcional de los AVA desarrollados para los cursos de lectoescritura y su uso tanto en el trabajo dirigido de la clase presencial como en el trabajo independiente del estudiante. 
El AVA está organizado en 8 secciones: 3 secciones de presentación del espacio académico, modelo educativo pedagógico, e interacción de propósito general; 3 secciones conformadas por tres módulos: gramática, lectura crítica y producción escrita con sus respectivos recursos didácticos y actividades complementarias; y 2 secciones destinadas para la evaluación diagnóstica y la

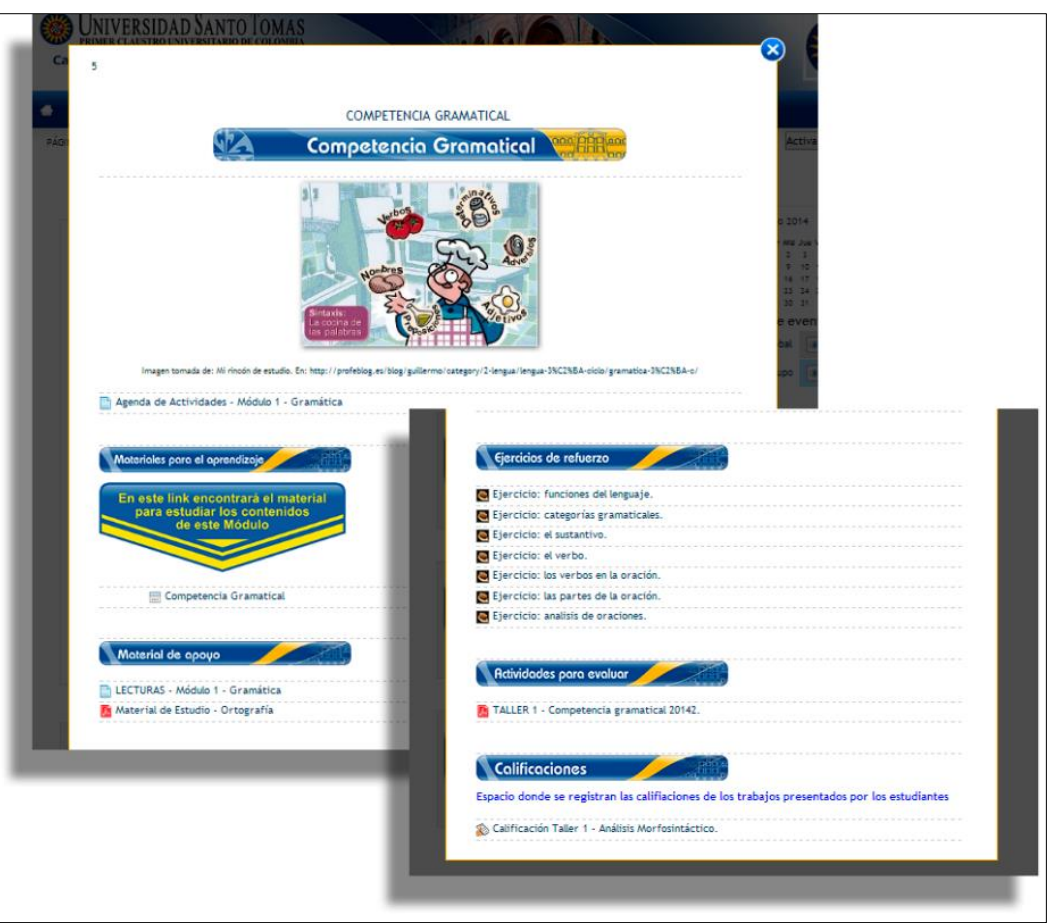
autoevaluación.

En el módulo1, donde se trabajan las competencias gramaticales o lingüísticas, se utilizan los AVA como herramienta de apoyo para las horas de clase presencial con el fin que el estudiante se familiarice con su uso; se lee el texto que se va a trabajar en clase; se realiza un ejercicio de modelación del análisis morfológico y sintáctico de un párrafo; se solucionan las dudas e interrogantes que surgen con respecto al tema tratado; y se proponen ejercicios de dificultad considerable que se desarrollan en compañía del docente con el fin que los estudiantes sigan paso a paso el ejemplo elaborado e interioricen el conocimiento, para que finalmente puedan ser evaluados mediante un taller de clase.

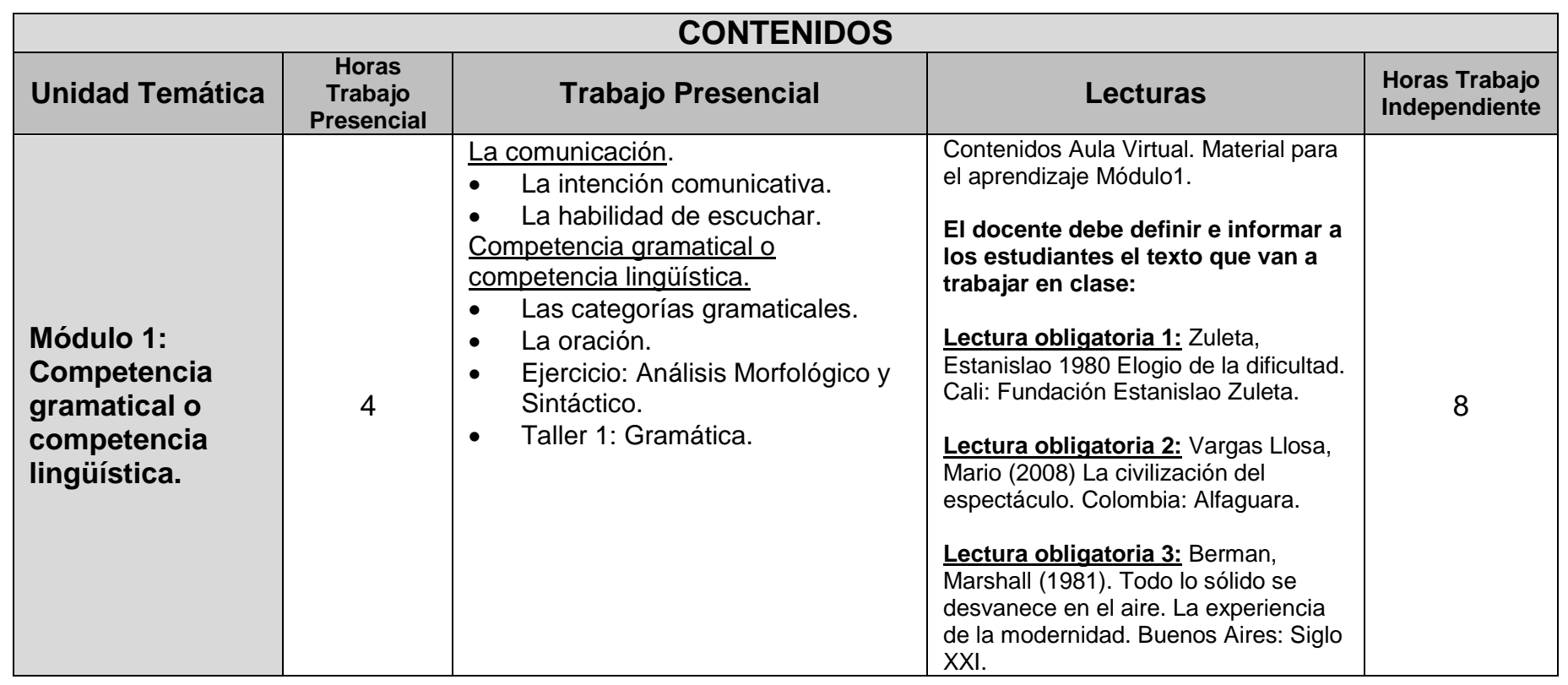

En las horas de trabajo independiente, el estudiante, que en clase identificó los temas que debe repasar o reforzar, debe ingresar al aula virtual estudiar en el material para el aprendizaje (OVA), desarrollar los ejercicios prácticos para que en la medida que va adquiriendo los conceptos los aplique en contexto; luego que ha estudiado el OVA encuentra materiales de apoyo que le permite complementar o profundizar lo aprendido, en este caso encuentra un recurso que le posibilita repasar algunas reglas ortográficas. Con el fin que se 


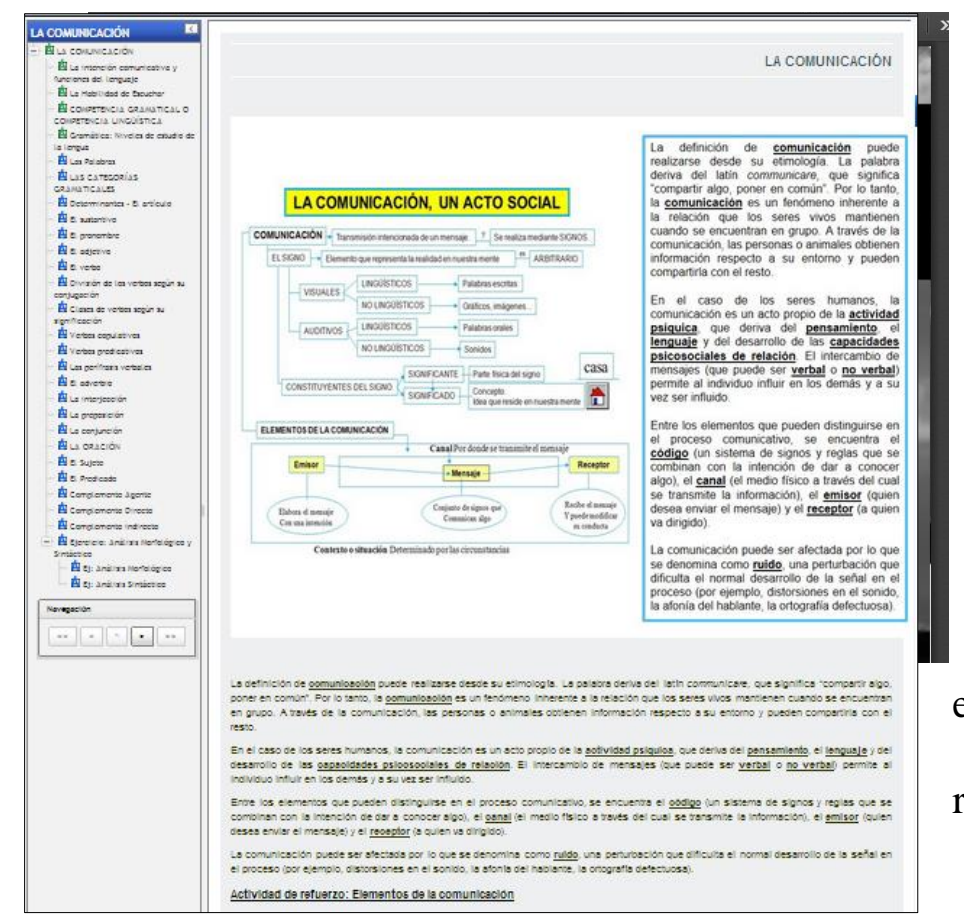

autoevalúe y determine si comprendió o requería volver a estudiar algún tema en específico se le proponen varios ejercicios de refuerzo

Durante la siguiente clase el docente solicita a los estudiantes que socialicen el trabajo realizado durante esas horas de Trabajo independiente con el objetivo de retroalimentar los ejercicios y despejar dudas. Para evaluar el proceso se aplica el taller 1, se socializa, se retroalimenta y se califica el desempeño de los estudiantes.

Los módulos 2 (lectura crítica) y 3 (producción escrita), aunque tiene la misma estructura del módulo1 - a saber, OVA, modelación de la competencia, ejercitación guiada por el docente y finalmente trabajo independiente del estudiante- profundizan más en la comprensión y análisis de textos específicos de su profesión y en la producción de textos argumentativos académicos que respondan a las necesidades propias de sus saberes profesionales. Además, en estos módulos los estudiantes adquieren técnicas de estudio como son el resumen, el cuadro sinóptico y el mapa conceptual, herramientas vitales para alcanzar un buen nivel de lectura crítica y de producción textual que trabajarán a lo largo de su vida académica.

Como se puede observar, en los cursos de lectoescritura de la USTA, los AVA están diseñados con el objetivo de articular los conceptos y destrezas aprendidas en las horas de trabajo presencial con las horas de trabajo independiente del estudiante, con el fin de que repase los preconceptos adquiridos en su etapa escolar, los relacione

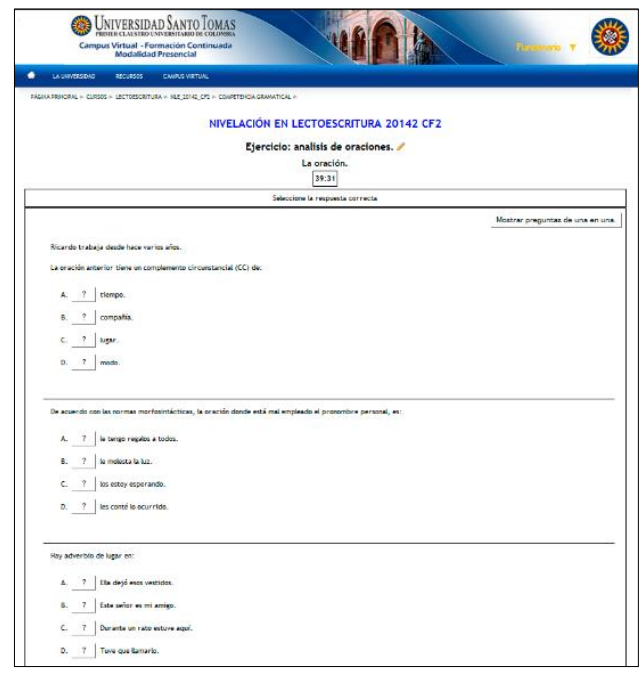
con los conceptos nuevos, refuerce aquellos que presentan alguna dificultad y los aplique en el contexto de la Educación Superior a través de ejercicios y lecturas especializadas.

\section{Una experiencia significativa}

La Representación Estudiantil de la Facultad de Ingeniería Electrónica presentó a la Decanatura la propuesta de elaborar una revista de estudiantes para conmemorar los 30 años de fundación de la Facultad. Al llevar esta propuesta a los estudiantes de primer semestre, surgió la idea de trabajar la redacción los artículos desde el curso de lectoescritura y asumir esta actividad como un proyecto de aula.

A partir de allí, se direccionó el curso a la producción de material para la revista y se conformó un comité editorial con los estudiantes para definir el tema central, ya que debía contar con algunos requisitos: (i) ser de 
actualidad, (ii) ser de interés general pero con énfasis en las temáticas propias de la ingeniería electrónica y (iii) ser novedoso para el lector. Basados en estas condiciones se definió que el tema de la revista sería "la tecnología implementada en el Mundial de Brasil 2014”, pues articulaba el propósito de la clase, la especificidad profesional y la pertinencia actual. Así, nació la primera edición de la Revista virtual "Bakelita" (http://electronica.usta.edu.co/index.php/revista-bakelita). Este proyecto concatenó el trabajo presencial del aula con el trabajo independiente de los estudiantes de lectoescritura a través del uso de los AVA.

\section{Caracterización de los cursos implementados en la oferta formativa de acompañamiento en}

\section{Lectoescritura}

Hasta este momento hemos explicado el uso de los AVA como herramientas de apoyo para el trabajo independiente de los estudiantes en los cursos de lectoescritura de los programas de la modalidad presencial. A continuación mostraremos cómo se articuló este proceso con la Oficina de Educación Virtual, qué herramientas se utilizaron para la realizar el seguimiento y análisis del uso de los AVA y los planes de mejora que se implementaron en la plataforma Moodle para optimizar la recolección de los datos, pues en el proceso de evaluación se encontró que algunos recursos no permitían un seguimiento adecuado por la forma en que se implementaron en el aula virtual.

En el primer semestre de 2013, el servicio de aulas virtuales para los cursos presenciales de lectoescritura se ofreció con la plataforma Moodle en su versión 1.9, y a partir del segundo semestre con la versión 2.3. Esto, sumado a las modificaciones del sistema de información que soporta el servicio Moodle (rediseño de módulos y cambios en la estructura de tablas en la base de datos), hizo difícil contrastar los resultados de seguimiento de la primera implementación con respecto a las ofrecidas a partir del segundo semestre de 2013.

\begin{tabular}{|c|l|c|c|}
\hline Periodo & \multicolumn{1}{|c|}{ AVA creados } & $\begin{array}{c}\text { Estudiantes } \\
\text { inscritos }\end{array}$ & $\begin{array}{c}\text { Estudiantes que } \\
\text { utilizan el AVA }\end{array}$ \\
\hline $2013-2$ & $\begin{array}{l}\text { 18 aulas para nivelación presencial, 1 } \\
\text { aula para nivelación virtual }\end{array}$ & 507 & 303 \\
\hline $2014-1$ & $\begin{array}{l}\text { 20 aulas para nivelación presencial, 1 } \\
\text { aula para nivelación virtual }\end{array}$ & 907 & 317 \\
\hline $2014-2$ & 17 aulas para nivelación presencial & 475 & 175 \\
\hline
\end{tabular}

En el primer semestre de 2013 se hizo uso del módulo de reportes disponible en la plataforma Moodle, y se generaron informes con los registros de actividad de los estudiantes participantes en los AVA. Con esta experiencia se evidenció que el servicio de reportes era funcional para los propósitos de seguimiento, pero su estructura dificultaba la obtención de datos organizados bajo la estructura que refleja la secuencia didáctica. Cada componente de esta secuencia quedó conformado por uno a más recursos y/o actividades, y en la estrategia de seguimiento se decidió unir los registros de acceso de los recursos didácticos integrados en cada etapa del trabajo independiente, esto con el fin de obtener los niveles de utilización de los recursos didácticos 
dispuestos en cada componente en lugar de la utilización de cada recurso por separado. El módulo de reportes se diseñó para presentar reportes de utilización de todas las actividades o por cada actividad en forma independiente.

La generación de reportes se enfocó entonces en la obtención de registros desde la base de datos Moodle y para esto se procedió a la instalación del módulo de reportes configurables que permitieran generar consultas personalizadas y adecuadas a las características de seguimiento planteadas anteriormente. Para obtener los registros de acceso, se desarrollaron dos script en lenguaje SQL que consultan cada recurso y/o actividad y lo agrupan con los demás recursos disponibles en cada etapa de la secuencia didáctica. De esta forma permitió obtener 5 datos por cada módulo: agenda del módulo, recursos SCORM, lecturas del módulo, actividades de refuerzo y actividades para evaluar.

En el segmento de código siguiente se aprecian los elementos que fueron tenidos en cuenta para consultar los registros de la base de datos:

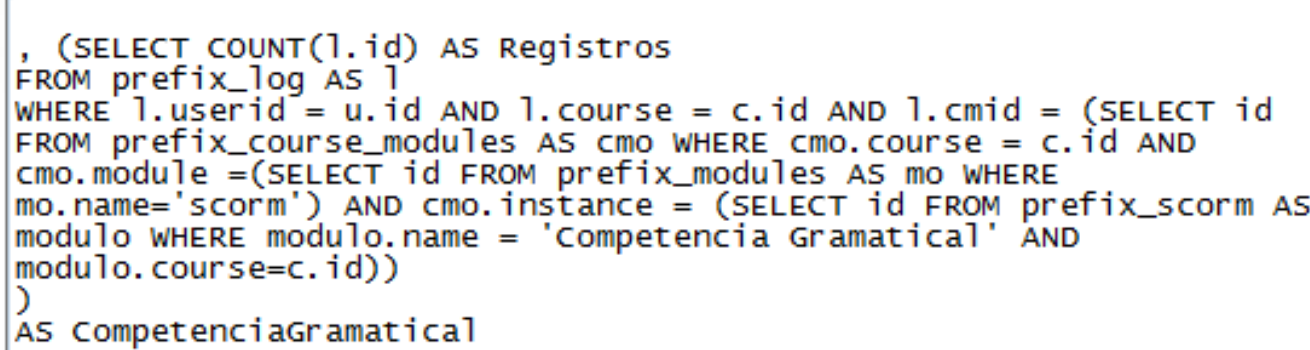

A continuación se presenta una muestra de los resultados de la consulta:

\begin{tabular}{|c|c|c|c|c|c|c|}
\hline $\begin{array}{l}\text { Periodo } \\
\text { Académic }^{-}\end{array}$ & Nombre del Curso & $\begin{array}{c}\text { Accesos Agenda } \\
\text { Módulo } 1\end{array}$ & $\begin{array}{c}\text { Accesos Recursos } \\
\text { SCORM }\end{array}$ & $\begin{array}{c}\text { Accesos Lecturas } \\
\text { Módulo } 1\end{array}$ & $\begin{array}{c}\text { Accesos Actividades } \\
\text { de Refuerzo }\end{array}$ & $\begin{array}{c}\text { Accesos Actividades } \\
\text { para Evaluar }\end{array}$ \\
\hline$\theta 20132$ & NIVELACION LECTO-ESCRITURA CFA & 27 & & 32 & 114 & 131 \\
\hline & NIVELACION LECTO-ESCRITURA CFB & 20 & 0 & 19 & 88 & 124 \\
\hline & NIVELACION LECTO-ESCRITURA CSA & 80 & 0 & 73 & 175 & 246 \\
\hline & NIVELACIÓN LECTO-ESCRITURA CSB & 25 & 0 & 19 & 33 & 81 \\
\hline & NIVELACION LECTO-ESCRITURA DGA & 36 & 0 & 16 & 193 & 77 \\
\hline & NIVELACIOON LECTO-ESCRITURA DGB & 30 & 0 & 25 & 67 & 101 \\
\hline & NIVELACION LECTO-ESCRITURA DRA & 67 & 0 & 25 & 38 & 46 \\
\hline & NIVELACION LECTO-ESCRITURA DRB & 62 & 0 & 31 & 59 & 81 \\
\hline & NIVELACIONN LECTO-ESCRITURA DRC & 19 & 0 & 18 & 50 & 40 \\
\hline & NIVELACION LECTO-ESCRITURA DRD & 55 & 0 & 34 & 101 & 157 \\
\hline & NIVELACIOON LECTO-ESCRITURAFTA & 42 & 0 & 21 & 73 & 75 \\
\hline & $\begin{array}{l}\text { NIVELACIÓN LECTO-ESCRITURA GOA } \\
\end{array}$ & 16 & 0 & 19 & 16 & 33 \\
\hline & NIVELACION LECTO-ESCRITURA GOB & 30 & 0 & 24 & 30 & 57 \\
\hline & NIVELACION LECTO-ESCRITURA PSA & 40 & 0 & 45 & 42 & 152 \\
\hline & NIVELACION LECTO-ESCRITURA PSB & 55 & 0 & 46 & 256 & 199 \\
\hline & NIVELACIOON LECTO-ESCRITURA REP & 4 & 0 & 2 & 65 & 12 \\
\hline & NIVELACIOON LECTO-ESCRITURA REP1 & $\frac{4}{1}$ & 0 & 1 & $\frac{0}{0}$ & $\frac{12}{1}$ \\
\hline & NIVELACIONN LECTO-ESCRITURA SOC & 23 & 0 & 10 & 120 & 34 \\
\hline$\nabla 20141$ & NIVELACIÖN EN LECTOESCRITURA 20141 CFA & 9 & 0 & 8 & 0 & 0 \\
\hline
\end{tabular}

Para contrastar la información de acceso de los tres semestres, se procedió a realizar adecuación de los datos de modo que se transformaron en porcentajes de utilización de los recursos y se agruparon las aulas por semestre con el fin de obtener el consolidado para los tres periodos. Las siguientes tablas presentan los resultados:

\begin{tabular}{|r|l|r|r|r|r|r|}
\hline $\begin{array}{l}\text { Periodo } \\
\text { Académico }\end{array}$ & Nombre del Curso & $\begin{array}{c}\text { Agenda } \\
\text { Módulo 1 }\end{array}$ & $\begin{array}{r}\text { Recursos } \\
\text { SCORM }\end{array}$ & $\begin{array}{r}\text { Lecturas } \\
\text { Módulo 1 }\end{array}$ & $\begin{array}{c}\text { Actividades } \\
\text { de Refuerzo }\end{array}$ & $\begin{array}{c}\text { Actividades } \\
\text { para Evaluar }\end{array}$ \\
\hline 20132 & NIVELACIÓN PRESENCIAL & $58,4 \%$ & $0,0 \%$ & $58,7 \%$ & $51,2 \%$ & $83,8 \%$ \\
\hline 20141 & NIVELACIÓN PRESENCIAL & $25,2 \%$ & $0,0 \%$ & $52,1 \%$ & $0,0 \%$ & $0,0 \%$ \\
\hline 20142 & NIVELACIÓN PRESENCIAL & $38,9 \%$ & $54,3 \%$ & $58,9 \%$ & $42,9 \%$ & $39,4 \%$ \\
\hline
\end{tabular}




\begin{tabular}{l|l|r|r|r|r|r|}
\hline $\begin{array}{l}\text { Periodo } \\
\text { Académico }\end{array}$ & Nombre del Curso & $\begin{array}{c}\text { Agenda } \\
\text { Módulo 2 }\end{array}$ & $\begin{array}{r}\text { Recursos } \\
\text { SCORM }\end{array}$ & $\begin{array}{r}\text { Lecturas } \\
\text { Módulo 2 }\end{array}$ & $\begin{array}{r}\text { Actividades } \\
\text { de Refuerzo }\end{array}$ & $\begin{array}{r}\text { Actividades } \\
\text { para Evaluar }\end{array}$ \\
\hline 20132 & NIVELACIÓN PRESENCIAL & $37,0 \%$ & $0,0 \%$ & $31,0 \%$ & $23,8 \%$ & $59,4 \%$ \\
\hline 20141 & NIVELACIÓN PRESENCIAL & $16,7 \%$ & $0,0 \%$ & $41,6 \%$ & $0,0 \%$ & $0,0 \%$ \\
\hline 20142 & NIVELACIÓN PRESENCIAL & $26,9 \%$ & $40,0 \%$ & $43,4 \%$ & $21,7 \%$ & $60,6 \%$ \\
\hline $\begin{array}{l}\text { Periodo } \\
\text { Académico }\end{array}$ & Nombre del Curso & Agenda & Recursos & Lecturas & $\begin{array}{r}\text { Actividades } \\
\text { de Refuerzo }\end{array}$ & $\begin{array}{c}\text { Actividades } \\
\text { para Evaluar }\end{array}$ \\
\hline 20132 & NIVELAClÓN PRESENCIAL & $21,8 \%$ & $0,0 \%$ & $8,6 \%$ & $13,9 \%$ & $54,5 \%$ \\
\hline 20141 & NIVELACIÓN PRESENCIAL & $5,0 \%$ & $0,0 \%$ & $7,9 \%$ & $8,5 \%$ & $0,0 \%$ \\
\hline 20142 & NIVELACIÓN PRESENCIAL & $7,4 \%$ & $16,0 \%$ & $12,0 \%$ & $8,0 \%$ & $26,9 \%$ \\
\hline
\end{tabular}

El seguimiento a las aulas virtuales creadas para el segundo semestre de 2013 y el primer semestre de 2014 no se pudo realizar ya que no fue posible obtener datos de acceso a los materiales para el aprendizaje (recursos SCORM), pues los OVA fueron almacenados en un repositorio de materiales didácticos multimedia implementando en la Universidad (disponible en http://mdm.usta.edu.co/), el cual no permitió generar la estadística de uso. Teniendo en cuenta esta dificultad, se montó en las aulas virtuales un acceso a cada material como enlaces vinculados con imágenes (etiquetas), y al realizar el seguimiento se encontró que este tipo de recursos no genera registros de accesos en la base de datos. Ante la imposibilidad de verificar el acceso a estos materiales, se decidió rediseñarlos y empaquetarlos al interior de las aulas con la actividad Paquete SCORM que permitió generar registros de acceso a cada página del material didáctico. En el seguimiento realizado a las aulas creadas para el segundo semestre de 2014, se pudo evidenciar que el $36.8 \%$ de los estudiantes hicieron usos de los materiales para el aprendizaje (recursos SCORM). Por otro lado se encontró que el 34.4\% de los estudiantes hizo uso de los materiales de apoyo (lecturas de los módulos).

\begin{tabular}{|c|c|c|c|c|c|c|c|c|c|c|c|c|c|c|}
\hline $\begin{array}{l}\text { Periodo } \\
\text { Académico }\end{array}$ & \multicolumn{2}{|c|}{ Nombre del Curso } & \multicolumn{2}{|c|}{$\begin{array}{l}\text { Accesos } \\
\text { Agenda }\end{array}$} & \multicolumn{2}{|c|}{\begin{tabular}{|l} 
Accesos \\
Recursos \\
SCORM
\end{tabular}} & \multicolumn{2}{|c|}{$\begin{array}{l}\text { Accesos } \\
\text { Lecturas }\end{array}$} & \multicolumn{2}{|c|}{\begin{tabular}{|l|} 
Accesos \\
Actividades \\
de Refuerzo
\end{tabular}} & \multicolumn{2}{|c|}{\begin{tabular}{|l|} 
Accesos \\
Actividades \\
para Evaluar
\end{tabular}} & \multicolumn{2}{|c|}{\begin{tabular}{|l} 
Total \\
estudiantes
\end{tabular}} \\
\hline 20132 & NIVEL & ACIÓN PRESENCIAL & & 118 & & 0 & & 99 & & 90 & & 200 & & 303 \\
\hline 20141 & NIVEL & ACIÓN PRESENCIAL & & 50 & & 0 & & 107 & & 9 & & 0 & & 317 \\
\hline 20142 & NIVEL & ACIÓN PRESENCIAL & & 43 & & 64 & & 67 & & 42 & & 74 & & 175 \\
\hline Total general & & & & 211 & & 64 & & 273 & & 141 & & 274 & & 795 \\
\hline $\begin{array}{l}\text { Period } \\
\text { Acadé }\end{array}$ & inico & Nombre del Curso & & $\begin{array}{l}\text { Accesc } \\
\text { Agend }\end{array}$ & & $\begin{array}{l}\text { Acces } \\
\text { Recur } \\
\text { SCOR }\end{array}$ & $\begin{array}{l}\text { Sos } \\
\text { sos } \\
\text { SM }\end{array}$ & $\begin{array}{l}\text { Accesc } \\
\text { Lectura }\end{array}$ & & $\begin{array}{l}\text { Acces } \\
\text { Activi } \\
\text { de Re }\end{array}$ & $\begin{array}{l}\text { sos } \\
\text { dades } \\
\text { fuerzo }\end{array}$ & $\begin{array}{l}\text { Acces } \\
\text { Activi } \\
\text { para E }\end{array}$ & $\begin{array}{l}\text { sos } \\
\text { dades } \\
\text { Evaluar }\end{array}$ & \\
\hline & 20132 & NIVELACIÓN PRESE & NCIAL & & $39,1 \%$ & & $0,0 \%$ & & $32,8 \%$ & & $29,6 \%$ & & $65,9 \%$ & \\
\hline & 20141 & NIVELACIÓN PRESE & NCIAL & & $15,7 \%$ & & $0,0 \%$ & & $33,9 \%$ & & $2,8 \%$ & & $0,0 \%$ & \\
\hline & 20142 & NIVELACIÓN PRESE & NCIAL & & $24,4 \%$ & & $36,8 \%$ & & $38,1 \%$ & & $24,2 \%$ & & $42,3 \%$ & \\
\hline Total g & general & & & & $26,5 \%$ & & $8,1 \%$ & & $34,4 \%$ & & $17,7 \%$ & & $34,4 \%$ & \\
\hline
\end{tabular}

Igualmente se encontró una tendencia en la disminución del acceso a recursos y actividades a medida que se desarrollaban los módulos. En los tres semestres el nivel de acceso a los recursos didácticos del módulo de Competencia Gramatical fue el mayor con registro del 36\% de utilización, seguido por los módulos de Lectura crítica con el 25\% y Producción Escrita con el 12\%.

\begin{tabular}{|c|c|c|c|c|c|c|c|c|c|c|}
\hline $\begin{array}{l}\text { Periodo } \\
\text { Académico }\end{array}$ & \multicolumn{2}{|c|}{ Nombre del Curso } & \multicolumn{2}{|c|}{ Módulo 1. Co } & \multicolumn{2}{|c|}{ Módulo 2. Led } & \multicolumn{2}{|c|}{ Módulo 3. Pro } & \multicolumn{2}{|c|}{$\begin{array}{l}\text { Total } \\
\text { estudiantes }\end{array}$} \\
\hline 20132 & NIVEL & ACIÓN PRESENCIAL & & 153 & & 92 & & 60 & & 303 \\
\hline 20141 & NIVELA & ACIÓN PRESENCIAL & & 49 & & 37 & & 14 & & 317 \\
\hline 20142 & NIVELA & ACIÓN PRESENCIAL & & 82 & & 67 & & 25 & & 175 \\
\hline Total general & & & & 284 & & 196 & & 98 & & 795 \\
\hline $\begin{array}{l}\text { Perioc } \\
\text { Acade }\end{array}$ & $\begin{array}{l}\text { do } \\
\text { émico }\end{array}$ & Nombre del Curso & & $\begin{array}{l}\text { Módulo } \\
\text { Compe } \\
\text { Grama }\end{array}$ & $\begin{array}{l}1 . \\
\text { tencia } \\
\text { tical }\end{array}$ & $\begin{array}{l}\text { Módulo } \\
\text { Lectura } \\
\text { Crítica }\end{array}$ & & $\begin{array}{l}\text { Módulo } \\
\text { Produc } \\
\text { Escrita }\end{array}$ & $\begin{array}{l}3 . \\
\text { ción }\end{array}$ & \\
\hline & 20132 & NIVELACIÓN PRESE & $\mathrm{NClAL}$ & & $50 \%$ & & $30 \%$ & & $20 \%$ & \\
\hline & 20141 & NIVELACIÓN PRESE & ENCIAL & & $15 \%$ & & $12 \%$ & & $4 \%$ & \\
\hline & 20142 & NIVELACIÓN PRESE & ENCIAL & & $47 \%$ & & $39 \%$ & & $14 \%$ & \\
\hline Total & general & & & & $36 \%$ & & $25 \%$ & & $12 \%$ & \\
\hline
\end{tabular}


El seguimiento de estos datos es significativo porque el docente puede analizar el uso de los AVA en el trabajo independiente de los estudiantes, pues le permite identificar los avances y dificultades en el proceso de aprendizaje de una manera personalizada. Por otra parte, también facilita la retroalimentación del trabajo presencial ya que tales avances y dificultades dinamizan la participación de los estudiantes en las clases.

\section{Conclusión}

A manera de síntesis, podemos afirmar que los AVA son un recurso al servicio del proceso de enseñanza y aprendizaje en la modalidad presencial de la Educación Superior. Sin embargo, su optimización requiere un cambio de paradigma organizacional, administrativo y tecnológico, que permita crear equipos de trabajo colaborativo articulados con las perspectivas académicas y pedagógicas expuestas en el PEI, el Modelo Pedagógico, el perfil de los egresados y los lineamientos curriculares de cada Institución, con el objetivo de dar sentido al uso de los AVA en el proceso de enseñanza y aprendizaje y motive a la innovación de herramientas didácticas que respondan a los retos que propone la sociedad del conocimiento.

Tras esta breve exposición y fundamentados en la experiencia que se ha proporcionado desde los cursos de lectoescritura en la USTA, podemos ratificar la idea de que la modalidad virtual no es opuesta a la presencial, pues el uso de ambientes virtuales de aprendizaje, como herramientas de apoyo para el trabajo independiente, enriquece y complementa los procesos educativos presenciales; no obstante, la comunidad académica y las instituciones de educación superior deben propender por promover las condiciones de posibilidad para su óptimo desarrollo, ya que su énfasis principal sigue orientado hacia la modalidad presencial tradicional.

En la actualidad, algunas Instituciones de Educación Superior no cuentan con los recursos tecnológicos adecuados para garantizar una conectividad eficiente, se trabaja con la limitación de software libre pues es poca la inversión que se hace en la adquisición de programas especializados, las salas de sistema son deficientes para la demanda de sus usuarios, etc. A este gran problema subyacen otras dificultades como las que enuncian Andrés Felipe Velásquez y Eduardo Augusto López Ramírez en su investigación, “Una mirada crítica al papel de las TIC en la educación superior en Colombia":

Entre los principales problemas que se presentan actualmente en la educación superior mediada por entornos virtuales de aprendizaje se encuentran:

1. Ausencia de estrategias didácticas en el diseño de material docente específico para uso en entornos virtuales de aprendizaje (Tradicionalmente los materiales son simples documentos elaborados en Word, Excel y Power Point diseñados para la educación presencial).

2. Bajo nivel de formación docente en el dominio de competencias sobre entornos virtuales de aprendizaje.

3. Falta de políticas y estrategias institucionales sobre la implementación y uso adecuado de los entornos virtuales de aprendizaje (Velásquez \& López Ramírez, 2010). 


\section{Referencias}

Cabero, J., \& Román , P. (2011). E-Actividades. Un referente básico para la formación en Internet. Sevilla: Aula Míltiple - Magisterio.

Galvis Panqueva, Á. (1998). Ambientes virtuales para participación en I sociedad del conocimiento. (UNIANDES-LIDIE, Ed.) Informática Educativa, 11(2), 247-260. Ministerio de Educación Nacional (MEN). (15 de marzo de 2012). Al día con las noticias. Monitoreo de prensa. Obtenido de Universitarios están mal en inglés y en comprensión de lectura: http://www.mineducacion.gov.co/observatorio/1722/article-300079.html

Ministerio de Educación Nacional. (10 de noviembre de 2001). Mineducación. Obtenido de Altablero: http://www.mineducacion.gov.co/1621/article-87727.html

Sunkel, G. (8 de julio de 2011). Red Latinoamericana Portal educativo. Obtenido de TIC para la educación en América Latina: hacia una perspectiva integral: http://www.relpe.org/especial-del-mes/tic-para-la-educacion-enamerica-latina-hacia-una-perspectiva-integral/

UNESCO. (9 de octubre de 1998). UNESCO Educación. Obtenido de declaracion mundial sobre la educacion superior en el siglo XXI: visión y acción y marco de acción prioritaria para el cambio y el desarrollo de la educación superior: http://www.unesco.org/education/educprog/wche/declaration_spa.htm

Velásquez , A. F., \& López Ramírez, E. A. (2010). Una mirada crítica al papel de las TIC en la educación superior. Obtenido de E-Mail Educativo, 1(1): http://www.revistas.unal.edu.co/index.php/email/article/view/12623 